\title{
Flight of Plasma Constitution Elements in a Cell
}

\author{
Masaaki Katoh, Yuji Matsubara , Takeo Ohte \\ Gunma National College of Technology, 580 Toriba-cho, Maebashi-shi, Gunma37I,Japan \\ mkato@elc.gunma-ct.ac.jp
}

Keywords: plasma improvement, flight patterns of plasma, plasma constituents, slit, cell

\section{Introduction}

Surface modification of various materials with plasma has been used widely and applied to many industrial fields [1] [2]. That can improve the material surface characteristics [3]. Treatment of three-dimensional materials and the high efficiency of treatment for a lot of materials are requested in plasma processing [4]. In a case of plasma processing, the plasma invades materials through narrow gaps and slits. The plasma components move though a narrow space (narrow space: space btween walls), and they are consumed in the meantime. The delivery of the plasma components onto the material surface of the narrow space has the problem that the modification is non-uniform.

The surface treatment processes a few materials in a very large quantity of plasma. However, as for the treatment in the space between walls, materials have a profound effect on plasma, and so an enough treatment effect cannot be expected because plasma itself changes.

However, the behavior and action of plasma in space inside a cell, has not been examined enough. Therefore a plasma treatment effect in a structural cell which can be invaded by plasma only from a slit is examined to get the behavior of plasma in the space between walls, namely the movement of plasma, change in quality, the effect to materials and soon.

Uniform treatment of the materials in the narrow space inside the cell and the mechanism which treat the materials in place far from the entrance are examined.

\section{Experimental method}

\subsection{Narrow space cell}

A narrow space cell is shown in Fig. 1.

Aluminum material was used for making the narrow space cell. The slit is $1 \mathrm{~mm}$ in height, $10 \mathrm{~mm}$ in width. The cell depth a is 8 [mm], 13 [mm], or 18 [mm]. Each cell is named cell I, cell II and cell III, respectively. The cell was shut by an aluminum plate tightly. The plasma can pass into the cell only though the slit.

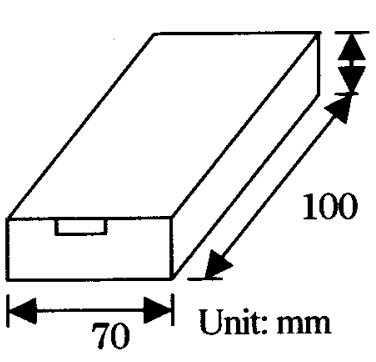

(a) cell

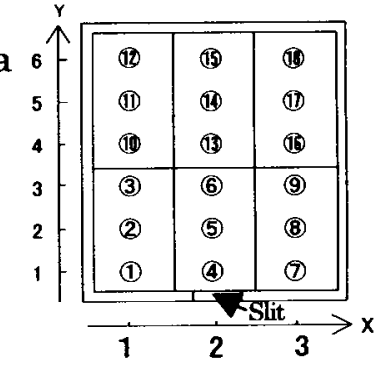

(b) Way of putting materials and measurement points of the contact angle of the water

Fig. 1 Narrow space cell and materials

\subsection{Materials}

Polypropylene (PP) was used as materials. The length of the sheet-like materials is $45 \mathrm{~mm}$, the width is $20 \mathrm{~mm}$ and the thickness is $1 \mathrm{~mm}$. They were washed by use of ethyl alcohol and water, and then dried before plasma treatment. The 6 sheets-like materials were set up in the narrow cell space. The contact angle of the water was measured at the 18 places.

\subsection{Plasma apparatus and generation of the plasma}

An induction type plasma generator in the external electrode formula (SAMCO ${ }^{\circledR}$ inc., Basic plasma kit model BP -1) was used for plasma treatment. Materials were put on the earth electrode inside the cell. The discharge chamber was evacuated by a rotary pump preliminarily for about 12 hours. The experimental condition was as follows. Treatment gas was $\mathrm{O}_{2}$ or $\mathrm{CF}_{4}$. Its pressure was $13.3 \mathrm{~Pa}$. The mass flow of gas was 
controlled in $15 \mathrm{ml} /$ minute. Then, a designated power (13.56 MHz) was supplied to the coil. R.F. discharge plasma was generated. And it was treated in a scheduled time.

\section{Result \& Discussion}

The contact angle of the material (PP) surfaces treated with $\mathrm{O}_{2}$ plasma became small, that is, the surfaces changed hydrophilic ally. If the difference (variation) of the contact angle before and after plasma treatment is big, the treatment effect by the plasma is high. In the case of $\mathrm{CF}_{4}$ plasma, contact angle became big and the material surfaces became hydrophobic

Fig. 2 and 3 show the difference of the contact angle with before and after plasma treatment. The treatment effect is big near to the slit of the cell and the effect is small far from the slit. The place plasma density is high near the slit and is low near the wall.

In the case of $\mathrm{O}_{2}$ plasma, it is not treated far from the slit when the treatment time is short. If the treatment time is lengthened, the whole region is treated.

In the case of $\mathrm{CF}_{4}$ plasma, the difference of the contact angle before and after plasma treatment was small, and the difference between the contact angles was small even if the treatment time was changed. When the depth of the cell was changed, the downward diffusion was examined. For the cells of $13 \mathrm{~mm}$ in depth (cell II) and $18 \mathrm{~mm}$ (cell III), the difference of the contact angles was measured before and after plasma treatment.

In the case of cell I, when the input power was increased, the change of the contact angle became big. Even in the case of cell II and cell III, it was the same as in cell $I$. When the input power grows big, the difference of the contact angle value tends to be in depend on the position of materials. For $\mathrm{O}_{2}$ plasma and $\mathrm{CF}_{4}$ plasma, the spatial distribution pattern of the treatment effect (the modification effect, and the extent of the plasma arrival) was similar.

The constituent elements of the $\mathrm{O}_{2}$ plasma are ions, radicals, electrons and molecules. The modification sources (particles producing the modification effect) are ions and radicals mainly. For the $\mathrm{CF}_{4}$ plasma the modification sources are similar, too. Ions of both plasma are reactive ions. Therefore the ions are affected by the electric field and the reactivity is high. The modification sources then react with the atoms of the material surface intensely. The neutral reactive radicals react with the atoms of the material surface, and oxygen atoms and fluorine atoms are introduced into the surface. It is considered that radicals extend by diffusion because the radicals are not affected by the electric field. The behavior and effect of modification sources which reach the surface and materials resemble for both the plasmas. Therefore it is considered that pattern of the modification effect resembles it.

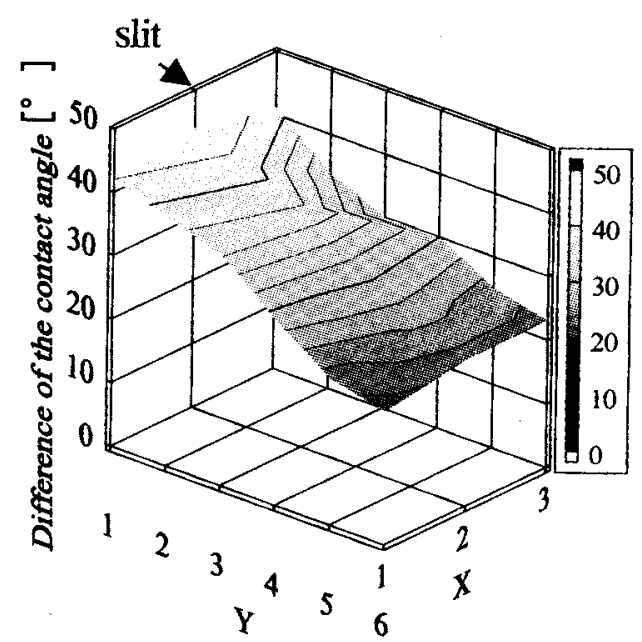

Fig.2 The spatial distribution of the treatment effect of the $\mathrm{O}_{2}$ plasma. (Treatment time 1 [min.])

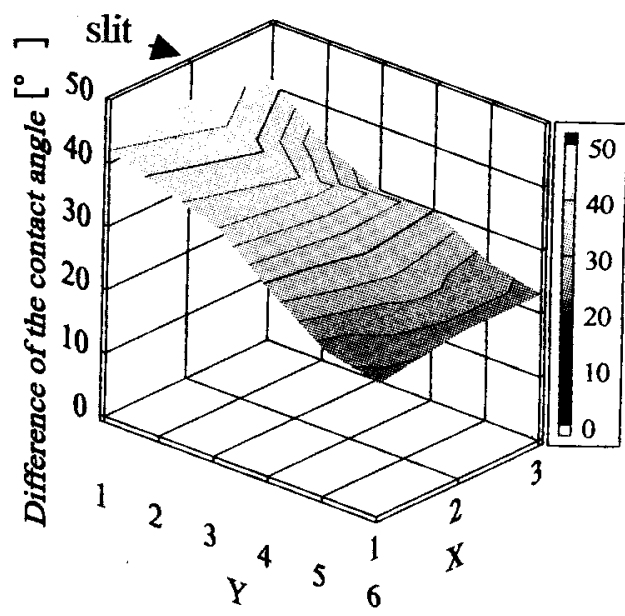

Fig.3 The spatial distribution of the treatment effect of the $\mathrm{CF}_{4}$ plasma. (Treatment time 5 [min.])

\section{References}

1. Brain Chapman, Glow Discharge processes, John Wiley \& Sons, Inc., (1980) p177.

2. M.Hudis, Techniques and Applications of Plasma Chemistry, edited by J.R.Hollahan, A. T. Bell, John Wiley \& Sons, Inc., (1974) pl13.

3. Y. Takada, J. Surf. Fin. Soc. Jpn., 47 (1996) 580 (in Japanese). 4. M. Katoh, M. Shibata, A. Kojima, T. Ohte, J. Photopoly. Sic.and Technol., 16 (2003) 